UNIVERSITATD BARCELONA

\section{Efficacy of conscious sedation for laser ambulatory ablation of benign thyroid}

\section{nodules}

CLÍNIC

Manuel López-Baamonde ${ }^{1}$, Felicia Hanzu², Mattia Squarcia ${ }^{3}$, Mireia Mora ${ }^{2}$, Ricard Valero ${ }^{1}$, Enrique Carrero ${ }^{1}$

\author{
${ }^{1}$ Anaesthesiology Department. Hospital Clínic. Universitat de Barcelona
}

2 Endocrinology Department. Hospital Clínic. Universitat de Barcelona

Background

${ }^{3}$ Department of Diagnostic Imaging. Hospital Clinic. Universitat de Barcelona

Laser ablation of the thyroid (LAT) is a new minimally invasive technique to treat thyroid benign nodules (TN).

It presents potential risks:

1. Tracheal perforation

2. Great vessel injury

Laryngeal oedema is the main risk in the immediate post-operative period

3. Cervical haematoma

In our hospital we perform LAT under conscious sedation in a prepared echography room with the patient in supine decubitus, cervical hyperextension and laser eyewear protection.

Sedation requirements: A. maintain spontaneous breathing, B. maintain patient's collaboration with response to verbal stimulus (Ramsay 2-3) ensuring C. highest comfort and adequate analgesia.

When the LAT begins oxygen administration must be suspended in order to minimize the ignition possibility and potential associated morbidity.

\title{
Objective
}

1) Value the efficacy and safety of conscious sedation for LAT ambulatory procedures in the first 25 cases between April 2014 and January 2016.

\section{Methods}
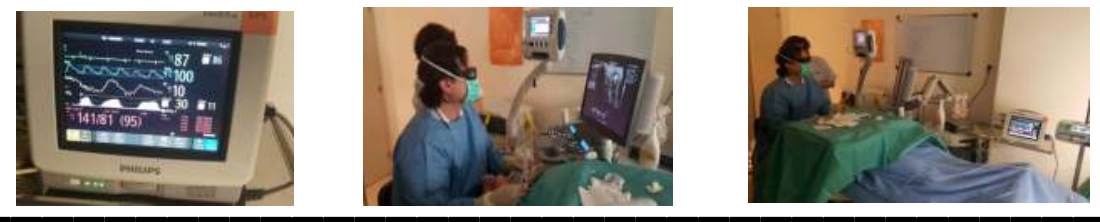

Monitoring: standard non-invasive monitoring (EKG, NIBP, SpO2, RR, EtCO2) and oxygen therapy with $2 \mathrm{~L}$ min nasal cannula.

Interventional technique: echo-guided 25 GA needle an a flexible optic fibre of $300 \mu \mathrm{m}$. NT $\leqq 10 \mathrm{~mL}$ are treated with $800 \mathrm{~J} / \mathrm{mL}$ and NT $>10 \mathrm{~mL}$ with $600 \mathrm{~J} / \mathrm{mL}$. Energy source: 2-5 W. Time: 5-10min. Result: central IV Premedication: pantoprazole $40 \mathrm{mg}$, ondansetron $4 \mathrm{mg}$, acetaminophen carbonization area contained in a coagulative necrosis zone.

$1 \mathrm{~g}$, metilprednisolone $0.5 \mathrm{mg} / \mathrm{Kg}$.

Recovery: $1 \mathrm{~h}$ in the radiology area box. Patient seated + cervical ice; echography control. Ambulatory procedure $\rightarrow$ Discharge.

Anaesthetic technique: local infiltration (mepivacaiine/lidocaiine) + propofol TCl (Target: Ramsay 2-3; 0.5-1 $\mathrm{mcg} / \mathrm{mL}$ ); IV ketamine (1st "rescue" option) and IV phentanyl (2nd "rescue" option).

\section{Results}

\begin{tabular}{|c|c|c|}
\hline \multicolumn{3}{|c|}{ Demographic Data } \\
\hline $\begin{array}{l}\text { Age, years } \\
\text { Weight }(\mathrm{Kg}) \\
\text { Height }(\mathrm{m}) \\
\text { Women, } \mathrm{n}(\%)\end{array}$ & & $\begin{array}{l}43 \pm 12 \\
76,9 \pm 18,3 \\
1,64 \pm 0,09 \\
18(72 \%)\end{array}$ \\
\hline ASA Classificatio & n (\%) & $\begin{array}{ll}\text { ASA I: } & 1(4 \%) \\
\text { ASA II: } & 15(60 \%) \\
\text { ASA III: } & 7(28 \%) \\
\text { ASA IV: } 0(0 \%) \\
\text { ASA V: } 0(0 \%)\end{array}$ \\
\hline Pathological Hist & & $\begin{array}{l}\text { HBP: } 12(48 \%) \\
\text { COPD:4 }(16 \%) \\
\text { OSA: } 3(12 \%) \\
\text { Cardiopathy:3(12 } \\
\%) \\
\text { DM: } 1(4 \%)\end{array}$ \\
\hline \multicolumn{3}{|c|}{ Drugs } \\
\hline Local Infiltration & $\begin{array}{l}\text { Mepi } \\
\text { Lidoc }\end{array}$ & $\begin{array}{ll}\text { ivacaine } & 22(88 \%) \\
\text { caine } & 1(4 \%)\end{array}$ \\
\hline Propofol TCI & $\begin{array}{l}\text { Time } \\
\text { Dose }\end{array}$ & $\begin{array}{l}60,4 \pm 27,6 \mathrm{~min} \\
: 125,3 \pm 76,6 \mathrm{mg}\end{array}$ \\
\hline $\begin{array}{l}\text { “Rescue” drug } \\
\text { association }\end{array}$ & $\begin{array}{l}\text { Ketan } \\
\text { Phen }\end{array}$ & $\begin{array}{rr}\text { mine } & 10(40 \%) \\
\text { tanyl } & 8(32 \%)\end{array}$ \\
\hline
\end{tabular}

\section{Conclusions}

\begin{tabular}{|ll|}
\hline \multicolumn{2}{|c|}{ Respiratory Depression } \\
\hline Apnoea ( $\geqq 10$ s) & $2(8 \%)$ \\
Bradipnoea (FR<10) & $5(20 \%)$ \\
Desaturation (SpO $2<90 \%)$ & $4(16 \%)$ \\
Ramsay 4 & $3(12 \%)$ \\
$\mathrm{CO}_{2}$ Re-inhalation & $1(4 \%)$ \\
Coughing & $1(4 \%)$ \\
\hline \multicolumn{1}{|c|}{ Early detection $\rightarrow$ Reversibility } \\
Verbal Stimulus & $5(20 \%)$ \\
Physical Stimulus & $1(4 \%)$ \\
FiO2 Rising & $0(0 \%)$ \\
Manual Airway Manoeuvres & $0(0 \%)$ \\
Oropharyngeal Cannula & $0(0 \%)$ \\
Ambu ${ }^{\circledR}$ & $0(0 \%)$ \\
Laryngeal Mask & $0(0 \%)$ \\
Intubation & $0(0 \%)$ \\
\hline
\end{tabular}

\begin{tabular}{|ll|}
\hline \multicolumn{2}{|c|}{ LAT Complications } \\
\hline Ignition & $0(0 \%)$ \\
Tracheal Perforation & $0(0 \%)$ \\
Oedema & $0(0 \%)$ \\
Stridency & $1(4 \%)$ \\
Perioral Paresthesia & $1(4 \%)$ \\
\hline
\end{tabular}

\begin{tabular}{|lc|}
\hline \multicolumn{2}{|c|}{ Postoperative Box Discharge Delay } \\
HBP & $1(4 \%)$ \\
\multirow{2}{*}{ Pain } & NRS 4-7: 3(12\%) \\
& NRS $>7: 3(12 \%)$ \\
\hline
\end{tabular}
every 8 hours and "rescue" with tramadol. Descendent corticoid prescription during 3 days. Clinical control in 7 days and ecography in 1

-Conscious sedation is an effective and safe techni que to perform laser ablation of beni gn thyroid nodules as an outpatient intervention.

-Monitoring and surveillance allow early detection and treatment of potential complications. 